\title{
Dynamic polarizabilities and related properties of clock states of ytterbium atom
}

\author{
V. A. Dzuba ${ }^{1}$ and A. Derevianko ${ }^{2}$ \\ ${ }^{1}$ School of Physics, University of New South Wales, Sydney, 2052, Australia \\ ${ }^{2}$ Physics Department, University of Nevada, Reno, Nevada 89557, USA
}

(Dated: August 17, 2009)

\begin{abstract}
We carry out relativistic many-body calculations of the static and dynamic dipole polarizabilities of the ground $6 s^{2}{ }^{1} S_{0}$ and the first excited $6 s 6 p^{3} P_{0}^{o}$ states of $\mathrm{Yb}$. With these polarizabilities, we compute several properties of $\mathrm{Yb}$ relevant to optical lattice clocks operating on the $6 s^{2}{ }^{1} S_{0}-6 s 6 p^{3} P_{0}^{o}$ transition. We determine (i) the first four magic wavelengths of the laser field for which the frequency of the clock transition is insensitive to the laser intensity. While the first magic wavelength is known, we predict the second, the third and the forth magic wavelengths to be $551 \mathrm{~nm}, 465 \mathrm{~nm}$, and $413 \mathrm{~nm}$. (ii) We reevaluate the effect of black-body radiation on the frequency of the clock transition, the resulting clock shift at $T=300 \mathrm{~K}$ being $-1.41(17) \mathrm{Hz}$. (iii) We compute long-range interatomic van der Waals coefficients (in a.u.) $C_{6}\left(6 s^{2}{ }^{1} S_{0}+6 s^{2}{ }^{1} S_{0}\right)=1909(160), C_{6}\left(6 s^{2}{ }^{1} S_{0}+6 s 6 p^{3} P_{0}\right)=2709(338)$, and $C_{6}\left(6 s 6 p^{3} P_{0}+6 s 6 p^{3} P_{0}\right)=3886(360)$. Finally, we determine the atom-wall interaction coefficients (in a.u.), $C_{3}\left(6 s^{2}{ }^{1} S_{0}\right)=3.34$ and $C_{3}\left(6 s 6 p^{3} P_{0}\right)=3.68$. We also address and resolve a disagreement between previous calculations of the static polarizability of the ground state.

PACS numbers: 06.30.Ft, 32.10.Dk, 31.25.-v
\end{abstract}

\section{INTRODUCTION}

Ytterbium atom is employed in a number of projects aimed at studying fundamental problems of modern physics by means of atomic physics. A large parity-violating signal on the $6 s^{2}{ }^{1} S_{0}-6 s 5 d{ }^{3} D_{1} 408$-nm transition of Yb has been recently observed at Berkeley [1]. A search for the CP-violating (T-, P- odd) permanent electric dipole moment of $\mathrm{Yb}$ was initiated [2]. A Bose-Einstein condensate in a dilute gas of the ground-state $\mathrm{Yb}$ atoms has been attained [3] and a number of experiments with ultracold $\mathrm{Yb}$ atoms has been carried out (see, e.g., Refs. [4, 5, [6]).

Here we focus on the optical lattice clock applications of $\mathrm{Yb}$. In the optical lattice clocks, neutral atoms are trapped in a standing wave of a laser light (optical lattice) operated at a certain "magic" wavelength 7]. At this wavelength a differential light perturbation of the two clock levels vanishes exactly. Using Yb atom in lattice clocks was proposed in Ref. [8] and experimentally demonstrated in Boulder 9, 10]. Apart from numerous technical applications, such atomic clocks may be used for searching for a variation of fundamental constants 11], mapping out atom-wall interaction [12], and serve as a basis for quantum information processing (see, e.g., 13, 14]).

In the present paper, we evaluate dynamic electric-dipole polarizabilities of the clock states, $6 s^{2}{ }^{1} S_{0}$ and $6 s 6 p^{3} P_{0}^{o}$. Dynamic polarizabilities of real and imaginary frequencies are linked to a variety of important atomic and molecular properties. In particular, we compute magic wavelengths, the effect of black-body radiation on the frequency of the clock transition, atom-wall interaction constants $C_{3}$, and, finally, long-range molecular van der Waals coefficients $C_{6}$ for the clock states. We take advantage of a close link between these properties and improve our theoretical predictions by referencing to measured properties, such as the first magic wavelength [9, 15] and the van der Waals coefficient for two ground-state atoms [5].

Ytterbium has been studied both experimentally (see, e.g., [5, 6, 9, 10, 15, 16, 17, 18]) and theoretically (see, e.g., [8, 19, 20, 21, 22, 23, 24, 25, 26]. Trapping and cooling schemes were discussed and implemented, the frequency of the clock transition was measured to a high accuracy [10], the first magic wavelength [9, 15] and the van der Waals coefficient for the ground state of $\mathrm{Yb}$ were also measured [5].

Theoretical studies are not free from a controversy. Calculated polarizability of the $\mathrm{Yb}$ ground state reported in Ref. [20] is in a disagreement with most of other ab initio or semi-empirical analyses. The source of this disagreement is explained below and the discrepancy is resolved. The resolution is closely related to the role of the excitations from the core which are discussed in detail. Corresponding statement can be formulated as a general theorem: Consider a second-order transition matrix element, involving summation over a complete set of intermediate states. Then, a contribution from a subspace spanned by degenerate states does not depend on mixing of these states. This statement has implications not only for computing polarizabilities, but also for the two-photon transition amplitudes, parity-violating amplitudes, etc. in $\mathrm{Yb}$ and other atoms. 


\section{METHOD OF CALCULATION}

In this section we start by recapitulating expressions for dynamic polarizability and its relations to magic wavelengths, and atom-atom and atom-wall interaction coefficients. Next we discuss our computational relativistic manybody method.

The ac Stark shift of an energy of a spherically-symmetric, $J=0$, atomic state $v$ in electro-magnetic wave of amplitude $\varepsilon$ and frequency $\omega$ is given by

$$
\Delta E_{v}(\omega)=-\alpha_{v}(\omega)\left(\frac{\varepsilon}{2}\right)^{2}
$$

where the electric-dipole dynamic polarizability reads (we use atomic units in which $\hbar=1,|e|=1$, and $m_{e}=1$ )

$$
\alpha_{v}(\omega)=\frac{2}{3} \sum_{k} \frac{E_{k}-E_{v}}{\left(E_{k}-E_{v}\right)^{2}-\omega^{2}}\langle v\|\mathbf{d}\| k\rangle^{2} .
$$

Here $\mathbf{d}$ is an electric dipole operator and summation is over a complete set of many-body states.

Magic laser frequency $\omega^{*}$ is found from the condition that the ac Stark shifts of both clock states $v$ and $w$ are identical so that the frequency of the transition does not depend on the laser intensity,

$$
\Delta E_{v}(\omega)-\Delta E_{w}(\omega)=-\left(\alpha_{v}(\omega)-\alpha_{w}(\omega)\right)\left(\frac{\varepsilon}{2}\right)^{2}=0
$$

Simply, at the magic frequencies, $\alpha_{v}\left(\omega^{*}\right)=\alpha_{w}\left(\omega^{*}\right)$.

Clock frequency shift due to black-body radiation is expressed in terms of the difference of static polarizabilities of the two clock levels (see detailed discussion in Ref. [21]).

We also require atomic polarizabilities evaluated at purely imaginary frequencies

$$
\alpha_{v}(i \omega)=\frac{2}{3} \sum_{k} \frac{E_{k}-E_{v}}{\left(E_{k}-E_{v}\right)^{2}+\omega^{2}}\langle v\|\mathbf{d}\| k\rangle^{2} .
$$

These are used in evaluating the atom-wall interaction constant $C_{3}[27]$

$$
C_{3}=\frac{1}{4 \pi} \int_{0}^{\infty} \alpha(i \omega) d \omega
$$

and the van der Waals coefficients $C_{6}$. For two separated atoms in states $w$ and $v$,

$$
C_{6}(w+v)=\frac{3}{\pi} \int_{0}^{\infty} \alpha_{w}(i \omega) \alpha_{v}(i \omega) d \omega
$$

The $C_{6}$ for two atoms interacting in identical states is subsumed by the above formula. Eq. (6) is derived under assumption that there are no downward dipole transitions from either $w$ or $v$ state, which holds in our case.

It is clear that for computing the polarizabilities, we need to determine electric dipoles and energies, and to perform the summation over a complete set of atomic states. To this end, we use a combination of the configuration-interaction (CI) method and the many-body perturbation theory (MBPT) to construct an effective Hamiltonian for two valence electrons (the CI+MBPT method [28, 29]). Further, we employ the Dalgarno-Lewis method [30] to reduce the summation over a complete set of many-electron states to solving an inhomogeneous system of linear equations.

\section{A. CI+MBPT method}

The ground state configuration of $\mathrm{Yb}$ is $4 f^{14} 6 s^{2}$ and most of the low-energy excited states correspond to configurations with one of the $6 s$ electrons being excited. All these states can be represented to a relatively high accuracy as states with two valence electrons above a closed-shell core. This is the starting point of our calculations: the computational (CI) model space is spanned by all possible excitations of the two valence electrons. Implications arising from the states with excitations from the outer $4 f$ core sub-shell will be discussed below.

The effective CI+MBPT Hamiltonian for two valence electrons has the form

$$
\hat{H}^{\mathrm{eff}}=\hat{h}_{1}\left(r_{1}\right)+\hat{h}_{1}\left(r_{2}\right)+\hat{h}_{2}\left(r_{1}, r_{2}\right)
$$


where $\hat{h}_{1}$ is the single-electron part of the relativistic Hamiltonian

$$
\hat{h}_{1}=c \hat{\alpha} \mathbf{p}+(\hat{\beta}-1) m_{e} c^{2}-\frac{Z e^{2}}{r}+V^{N-2}+\hat{\Sigma}_{1}
$$

and $\hat{h}_{2}$ is the two-electron part of the Hamiltonian

$$
\hat{h}_{2}\left(r_{1}, r_{2}\right)=\frac{e^{2}}{\left|\mathbf{r}_{1}-\mathbf{r}_{2}\right|}+\hat{\Sigma}_{2}\left(r_{1}, r_{2}\right) .
$$

In these equations, $\hat{\alpha}$ and $\hat{\beta}$ are the conventional Dirac matrices, $V^{N-2}$ is the Dirac-Hartree-Fock (DHF) potential of the closed-shell atomic core $(N-2=68, Z=70)$, and $\hat{\Sigma}$ is the correlation operator. It represents terms in the Hamiltonian arising due to virtual excitations from atomic core (see Ref. [28, 29] for details). $\hat{\Sigma} \equiv 0$ corresponds to the standard CI method. $\hat{\Sigma}_{1}$ is a single-electron operator. It represents a correlation interaction (core-polarization) of a particular valence electron with the atomic core. $\hat{\Sigma}_{2}$ is a two-electron operator. It represents screening of the Coulomb interaction between the two valence electrons by the core electrons. We calculate $\hat{\Sigma}$ in the second order of the MBPT. We use a B-spline technique [31] to construct a complete set of single-electron orbitals. We use 40 B-splines in a cavity of radius $R=40 a_{B}$ and calculate the eigenstates of the $V^{N-2}$ DHF Hamiltonian up to the maximum value of the angular momentum $l_{\max }=5$. The same basis is used in computing $\hat{\Sigma}$ and in constructing the two-electron states for the valence electrons. 30 out of 40 lowest-energy states for every $l$ up to $l_{\max }=5$ are used to calculate $\hat{\Sigma}$ and 14 lowest states above the core are used for every $l$ up to $l_{\max }=4$ to construct the two-electron states.

The two-electron valence states are found by solving the eigenvalue problem,

$$
\hat{H}^{\mathrm{eff}} \Psi_{v}=E_{v} \Psi_{v},
$$

using the standard CI techniques. Calculated and experimental energies of a few lowest-energy states of $\mathrm{Yb}$ are presented in Table I. One can see that the pure $a b$ initio energies are already close to the experimental values. However, for improving the accuracy further, we re-scale the correlation operator $\hat{\Sigma}_{1}$ by replacing $\hat{\Sigma}_{1}$ in the effective Hamiltonian (77) in each partial wave $s, p_{1 / 2}, p_{3 / 2}, \ldots$ by $f_{a} \hat{\Sigma}_{1}$. The rescaling factors are $f_{s}=0.8772, f_{p}=1.03$, $f_{d}=0.933$, and $f_{f}=1$. These values are chosen to fit the experimental spectrum of Yb. The result of the fitting is shown in Table \. Note that the fitting is not exact for all the levels because the number of levels is larger than the

\begin{tabular}{|c|c|c|c|c|c|}
\hline \multicolumn{2}{|c|}{ State } & \multicolumn{2}{|c|}{$J \quad$ Exp. 32$]$} & \multicolumn{2}{|c|}{$\begin{array}{c}\text { CI+MBPT } \\
a b \text { initio Rescaled } \hat{\Sigma}_{1}\end{array}$} \\
\hline $4 f^{14} 6 s^{2}$ & ${ }^{1} \mathrm{~S}$ & 0 & 0.0 & 0.0 & 0.0 \\
\hline \multirow[t]{3}{*}{$4 f^{14} 6 s 6 p$} & ${ }^{3} \mathrm{P}^{o}$ & 0 & 17288 & 18246 & 17289 \\
\hline & & 1 & 17992 & 18946 & 17996 \\
\hline & & 2 & 19710 & 20688 & 19759 \\
\hline \multirow[t]{3}{*}{$4 f^{14} 5 d 6 s$} & ${ }^{3} \mathrm{D}$ & 1 & 24489 & 24922 & 24489 \\
\hline & & 2 & 24752 & 25195 & 24743 \\
\hline & & 3 & 25271 & 25765 & 25276 \\
\hline $4 f^{14} 6 s 6 p$ & ${ }^{1} \mathrm{P}^{o}$ & 1 & 25068 & 26463 & 25611 \\
\hline $4 f^{13} 5 d 6 s^{2}$ & $7 / 2,5 / 2)^{\circ}$ & 1 & 28857 & & \\
\hline $4 f^{14} 5 d 6 s$ & ${ }^{1} \mathrm{D}$ & 2 & 27678 & 28485 & 27812 \\
\hline $4 f^{14} 6 s 7 s$ & ${ }^{3} \mathrm{~S}$ & 1 & 32695 & 33262 & 32668 \\
\hline $4 f^{14} 6 s 7 s$ & ${ }^{1} \mathrm{~S}$ & 0 & 34351 & 34871 & 34280 \\
\hline
\end{tabular}
number of fitting parameters.

TABLE I: Energy levels of $\mathrm{Yb}\left(\mathrm{cm}^{-1}\right)$. The $4 f^{13} 5 d 6 s^{2}(7 / 2,5 / 2)_{1}^{o}$ level lies outside of the computational model space.

Transition amplitudes are found with the random-phase approximation (RPA) [33, 34]

$$
E 1_{v w}=\left\langle\Psi_{v}\left\|d_{z}+\delta V^{N-2}\right\| \Psi_{w}\right\rangle,
$$

where $\delta V^{N-2}$ is the correction to the core potential due to core polarization by an external electric field. We compile our representative dipole matrix elements in Table I. The values were computed in the CI+MBPT approach. We also compare with a high-precision result [16] derived from fitting molecular long-range potentials to photoassociation spectra taken with ultracold samples. The $16 \%$ theory-experiment disagreement for the $6 s^{2}{ }^{1} S_{0}-6 s 6 p{ }^{1} P_{1}^{o}$ amplitude is due to mixing of the $6 s 6 p{ }^{1} P_{1}^{o}$ state with the core-excited state $4 f^{13} 5 d 6 s^{2}(7 / 2,5 / 2)_{1}^{o}$, which lies outside the computational model space (see Section [II]) 
TABLE II: Reduced matrix elements (a.u.) of the electric dipole operator for transitions in Yb. Values were computed in the CI+MBPT approach. The theory-experiment disagreement for the $6 s^{2}{ }^{1} S_{0}-6 s 6 p^{1} P_{1}^{o}$ is due to mixing of the $6 s 6 p{ }^{1} P_{1}^{o}$ state with the core-excited state $4 f^{13} 5 d 6 s^{2}(7 / 2,5 / 2)_{1}^{o}$, which lies outside the computational model space (see Section III)

\begin{tabular}{lll}
\hline \hline Transition & Calc. & Exp. \\
\hline $6 s^{2}{ }^{1} S_{0}-6 s 6 p^{3} P_{1}^{o}$ & 0.587 \\
$6 s^{2}{ }^{1} S_{0}-6 s 6 p^{1} P_{1}^{o}$ & 4.825 \\
$6 s 6 p^{3} P_{0}^{o}-5 d 6 s^{3} D_{1}$ & 2.911 \\
$6 s 6 p^{3} P_{0}^{o}-6 s 7 s{ }^{1} S_{0}$ & 1.952 \\
\hline \hline
\end{tabular}

${ }^{a}$ Ref. [16]

\section{B. Dalgarno-Lewis and RPA methods}

Computing polarizability requires summing over a complete set of many-body states. The CI+MBPT subspace of two valence electrons covers only a part of this set. The other class of intermediate state are core excitations, where the valence electrons serve as spectators. Notice, that even in the independent particle picture, the state of the valence electrons affects possible core excitations: core electrons can not be promoted into excited orbitals occupied by the valence electrons due to the Pauli exclusion principle. Following this discussion, we divide the polarizability into three parts [27],

$$
\alpha(\omega)=\alpha_{\text {val }}(\omega)+\alpha_{\text {core }}(\omega)+\alpha_{\text {core-val }}(\omega) .
$$

The valence contribution, $\alpha_{\text {val }}(\omega)$, is given by (2) where $v$ and $k$ are two-electron CI states (i.e., $k$ lie entirely in the model space). We use the Dalgarno-Lewis method [30] for summing over a complete set of two-electron states. In this method, a correction $\delta \Psi_{v}$ to the two-electron wave function of the state $v$ is introduced and the contribution of the valence electrons to the polarizability is expressed as (here we consider static polarizability)

$$
\alpha_{\mathrm{val}}(0)=\frac{2}{3}\left\langle\delta \Psi_{v}\|\mathbf{d}\| \Psi_{v}\right\rangle
$$

The correction $\delta \Psi_{v}$ is found by solving the system of linear inhomogeneous equations

$$
\left(\hat{H}^{\mathrm{eff}}-E_{v}\right) \delta \Psi_{v}=-\left(d_{z}+\delta V^{N-2}\right) \Psi_{v}
$$

In case of dynamic polarizabilities we employ the identity

$$
\frac{E_{k}-E_{v}}{\left(E_{k}-E_{v}\right)^{2}-\omega^{2}}=\frac{1}{\left(E_{k}-E_{v}\right)-\omega}+\frac{1}{\left(E_{k}-E_{v}\right)+\omega},
$$

i.e., the equations (13) need to be solved twice at two different energies $E=E_{v} \pm \omega$. For polarizabilities of purely imaginary argument, $\omega \rightarrow i \omega$ in the above equations.

The core polarizability is found using the RPA method 35] (linearized response theory). This method yields required eigen-energies of many-body particle-hole excitations from the core and relevant electric-dipole transition amplitudes. The dynamic core polarizability reads

$$
\alpha_{\text {core }}(i \omega)=\sum_{\omega_{\mu}>0} \frac{f_{\mu}}{\left(\omega_{\mu}\right)^{2}+\omega^{2}} .
$$

Here the summation is over particle-hole excitations from the ground state of the atomic core; $\omega_{\mu}$ are excitation energies and $f_{\mu}$ are the corresponding electric-dipole oscillator strengths. Technically, compared to the original, differential-equation method of Ref. [35], we use a B-spline basis set expansion. An important property of the RPA core polarizability is that it satisfies (non-relativistically) the Thomas-Reiche-Kuhn sum rule, $\lim _{\omega \rightarrow \infty} \omega^{2} \alpha_{\text {core }}(i \omega)=N-2$. This property is especially important in evaluating the $C_{3}$ and $C_{6}$ coefficients for heavy atoms [27].

Finally, the core-valence counter term, $\alpha_{\text {core-val }}(\omega)$, is small. At the DHF level, we find that the relevant contributions to static polarizabilities are below $1 \%$ and we neglect it at our present level of accuracy.

\section{THE ROLE OF THE $4 f^{14} 6 s 6 p^{1} P_{1}^{o}-4 f^{13} 5 d 6 s^{2}(7 / 2,5 / 2)_{1}^{o}$ MIXING}

There is a number of relatively low-lying states in the discrete spectrum of Yb which have an excitation from the outer $4 f$ sub-shell of the core. These states certainly cannot be considered as states with two valence electrons above 
closed shells. One of such states is $4 f^{13} 5 d 6 s^{2}(7 / 2,5 / 2)_{1}^{o}$ which lies close to the $4 f^{14} 6 s 6 p{ }^{1} P_{1}^{o}$ state (see Table 【). Notice that both states have the same total angular momentum and parity. Therefore the Coulomb interaction strongly mixes the two states. This mixing complicates the calculations. Our effective Hamiltonian (7) does take into account excitations from the core via second-order correlation operator $\hat{\Sigma}$. Yet, the second-order approximation is not adequate in this particular case, as the two states are separated by mere 0.0173 a.u. $\left(3789 \mathrm{~cm}^{-1}\right)$.

The calculation of the ground state polarizability is affected by the $4 f^{14} 6 s 6 p^{1} P_{1}^{o}-4 f^{13} 5 d 6 s^{2} 6(7 / 2,5 / 2)_{1}^{o}$ mixing. The $6 s^{2}{ }^{1} \mathrm{~S}_{0} \rightarrow 6 s 6 p{ }^{1} \mathrm{P}_{1}^{o}$ electric dipole transition contributes about $90 \%$ to the polarizability of the ground state. Corresponding theoretical CI+MBPT transition amplitude, 4.825 a.u., deviates significantly from the experimental value 4.148 a.u. [16]. As it was pointed out in Ref. [19] the reason for this disagreement is the above mentioned mixing.

Calculation of the core polarizability displays a similar dependence on the mixing. Our RPA result for $\alpha_{\text {core }}(0)=$ 6.39 a.u. At the same time, semi-empirical result 24] based on the life-time measurements of low-lying core-excited states in $\mathrm{Yb}$ suggests a much higher value of 24(4) a.u. Again, the reason for this large difference lies in the strong mixing of the $4 f^{13} 5 d 6 s^{2}(7 / 2,5 / 2)_{1}^{o}$ and $4 f^{14} 6 s 6 p{ }^{1} P_{1}^{o}$ states. Indeed, in the RPA core polarizability calculations, we consider a different system, $\mathrm{Yb}^{++}$, which is unaware of the presence of the two valence electrons.

Since the $6 s^{2}{ }^{1} \mathrm{~S}_{0} \rightarrow 6 s 6 p{ }^{1} \mathrm{P}_{1}^{o}$ transition contributes about $90 \%$ to the polarizability of the ground state, the authors of Ref. [20] suggested to replace the CI+MBPT dipole matrix element by its experimental value. This was done to improve the accuracy of computing the polarizability. Below we argue that such a substitution cannot be justified unless a similar correction is done for the $4 f_{7 / 2} \rightarrow 5 d_{5 / 2}$ transition amplitude in the calculation of the core polarizability.

Let us use the following notation for the pure (unmixed) states

$$
\begin{aligned}
& \Phi_{1}=\left|4 f^{14} 6 s 6 p^{1} \mathrm{P}_{1}^{o}\right\rangle, \\
& \Phi_{2}=\left|4 f^{13} 5 d 6 s^{2}(7 / 2,5 / 2)_{1}^{o}\right\rangle .
\end{aligned}
$$

The mixed states can be written as

$$
\begin{aligned}
& \Psi_{1}=\Phi_{1} \cos \phi-\Phi_{2} \sin \phi, \\
& \Psi_{2}=\Phi_{1} \sin \phi+\Phi_{2} \cos \phi,
\end{aligned}
$$

where $\phi$ is mixing angle.

Let also use $\Psi_{0} \equiv \Phi_{0}$ for the ground state wave function.

If we neglect a small energy difference between the states $\Psi_{1}$ and $\Psi_{2}$ (the relevant energy denominators entering $\alpha(0)$ differ by about $10 \%)$ then their total contribution to the polarizability of the ground state is proportional to

$$
\begin{aligned}
& \left\langle\Psi_{0}\|\mathbf{d}\| \Psi_{1}\right\rangle^{2}+\left\langle\Psi_{0}\|\mathbf{d}\| \Psi_{2}\right\rangle^{2}= \\
& \left\langle\Phi_{0}\|\mathbf{d}\| \Phi_{1}\right\rangle^{2}+\left\langle\Phi_{0}\|\mathbf{d}\| \Phi_{2}\right\rangle^{2}
\end{aligned}
$$

and does not depend on the mixing angle $\phi$. This means that either bases $\left\{\Phi_{0}, \Phi_{1}, \Phi_{2}\right\}$ or $\left\{\Psi_{0}, \Psi_{1}, \Psi_{2}\right\}$ can be used in the calculations. The ab initio calculations within the CI+MBPT framework in which Yb is considered as a twovalence-electrons atom uses the non-mixed basis while the analysis based on experimental data naturally corresponds to the use of the mixed basis. Both approaches produce close results as will be demonstrated in Section $\mathrm{V}$. By contrast, the substitution of the experimental value for the $\left\langle 6 s^{2}{ }^{1} \mathrm{~S}_{0}|\mathbf{D}| 6 s 6{ }^{1} \mathrm{P}_{1}^{o}\right\rangle$ transition amplitude is equivalent to using the mixed non-orthogonal basis $\left\{\Psi_{0}, \Psi_{1}, \Phi_{2}\right\}$. This seems to introduce a large error into the calculation of the polarizability. Indeed, the value reported in Ref. [20,21] is 111.3 a.u. which is about $30 \%$ smaller than the results of most ab initio calculations and semi-empirical analyses, compiled in Table III]. For example, the analysis [24] of the experimental data yielded $\alpha_{\mathrm{S}_{0}}(0)=136.4(4.0)$ a.u.

The result of Ref. [20, 21] is brought into an agreement with that of Ref. 24] by consistently correcting the core polarizability for the mixing. A DHF contribution from the $4 f$ subshell to core polarizability is 1.9 a.u. If we replace this value by the value [24] based on experimental data (i.e., account for the mixing), 24(4) then the result of Ref. 20] increases to $\alpha_{1} \mathrm{~S}_{0}(0)=133$ a.u. consistent with the value of 136.4(4.0) a.u. from Ref. [24].

It is useful to find out what happens if the difference in energies of the two mixed states is not neglected. We will use a short-hand notation $\Delta E_{1}=E\left(\Psi_{1}\right)-E\left(\Psi_{0}\right), \Delta E_{2}=E\left(\Psi_{2}\right)-E\left(\Psi_{0}\right)$, and $\delta=1 / \Delta E_{2}-1 / \Delta E_{1}$. Since the state $\Psi_{2} \equiv 4 f^{13} 5 d 6 s^{2} 6(7 / 2,5 / 2)_{1}^{o}$ is above the state $\Psi_{1} \equiv 4 f^{14} 6 s 6 p{ }^{1} P_{1}^{o}$ the parameter $\delta$ is negative. Further, $A_{01}=\left\langle\Phi_{0}\|\mathbf{d}\| \Phi_{1}\right\rangle$ and $A_{02}=\left\langle\Phi_{0}\|\mathbf{d}\| \Phi_{2}\right\rangle$.

A partial contribution of the two states into the polarizability reads

$$
\delta \alpha=\frac{1}{\Delta E_{1}}\left(A_{01}^{2}+A_{02}^{2}\right)+\delta\left(A_{01} \sin \phi+A_{02} \cos \phi\right)^{2} .
$$


We see that when the core-excited states lie above the states with which they are mixed, the correction to the polarizability due to this mixing is negative. This is the case for both polarizabilities of Yb considered in this work. We would keep this in mind while analyzing the accuracy of the calculations in Section $\mathrm{V}$.

\section{MIXING OF DEGENERATE STATES}

In the previous section we considered a particular case of mixing of the $4 f^{14} 6 s 6 p^{1} P_{1}^{o}$ and $4 f^{13} 5 d 6 s^{2} 6(7 / 2,5 / 2)_{1}^{o}$ states of $\mathrm{Yb}$ and demonstrated that this mixing can be ignored in the $a b$ initio calculations of the ground-state polarizability of $\mathrm{Yb}$. Notice that similar problems arise in computing the polarizability of the ${ }^{3} \mathrm{P}_{0}^{o}$ state and in many other cases not limited to calculations of the polarizabilities of $\mathrm{Yb}$. Therefore, it may be useful to treat a general case and formulate the following theorem: Consider a second-order transition amplitude, involving summation over a complete set of intermediate states. Then, a contribution from a subspace spanned by degenerate states does not depend on mixing of these states.

A generic contribution to a second-order transition amplitude can be written as (here $E_{0}$ is some energy off-set)

$$
A_{i f}=\sum_{m} \frac{\left\langle F_{f}|\hat{P}| F_{m}\right\rangle\left\langle F_{m}|\hat{Q}| F_{i}\right\rangle}{E_{0}-E_{m}}
$$

Here $F_{i}$ and $F_{f}$ are some initial and final atomic states, and $\hat{P}$ and $\hat{Q}$ are some operators. Let's assume that the summation over the complete set of intermediate states $F_{m}$ subsumes a summation over a group of degenerate states $\mathcal{D}$ and rewrite the corresponding partial contribution as

$$
\Delta A_{i f}=\frac{1}{\Delta E} \sum_{m \in \mathcal{D}}\left\langle F_{f}|\hat{P}| F_{m}\right\rangle\left\langle F_{m}|\hat{Q}| F_{i}\right\rangle .
$$

Here the summation ranges only over degenerate states and $\Delta E$ is their common energy denominator.

As in the previous section, we introduce the pure non-mixed basis states $\Phi_{k}$ and rewrite the real (mixed) states $F_{m}$ as an expansion

$$
F_{m}=\sum_{k} c_{m k} \Phi_{k}
$$

Both sets $\Phi$ and $F$ can be made orthonormal, with the matrix $C=\left\{c_{m k}\right\}$ being a unitary matrix, i.e., $C C^{\dagger}=I$. Substitution of (19) into (18) leads to

$$
\begin{aligned}
\Delta A_{i f} & =\frac{1}{\Delta E} \sum_{k l}\left\langle F_{f}|\hat{P}| \Phi_{k}\right\rangle\left\langle\Phi_{l}|\hat{Q}| F_{i}\right\rangle \sum_{m} c_{m k} c_{m l}^{*} \\
& =\frac{1}{\Delta E} \sum_{k}\left\langle F_{f}|\hat{P}| \Phi_{k}\right\rangle\left\langle\Phi_{k}|\hat{Q}| F_{i}\right\rangle .
\end{aligned}
$$

Here we used the ortho-normality condition, $\sum_{k} c_{k m} c_{k l}^{*}=\delta_{m l}$. We see that the final expression does not depend on the mixing coefficients $c$.

We stress two conditions under which this theorem is valid. It is assumed that the unmixed states possess the same energy and that there is no mixing with the states outside the degenerate subspace. These two conditions are probably never fulfilled exactly. However, there is a large number of cases when they are fulfilled approximately. One of the criteria is that the average energy spread in the quasi-degenerate subspace $\left|\delta E_{\mathcal{D}}\right| \ll\left|E_{0}-\bar{E}_{\mathcal{D}}\right|$, where $\bar{E}_{\mathcal{D}}$ is the average energy in the subspace.

Why is this practically important? In $\mathrm{Yb}$, to rigorously account for the mixing, one has to extend the twovalence-electron model space to include computationally-expensive core-excited states. The theorem claims that while computing polarizabilities, a much smaller two-valence-electron space would suffice. In other words, at the CI stage, the excitations from the $4 f$ subshell can be safely ignored (even) if there are nearby two-electron states of the same symmetry. (Of course, virtual core excitations are included in the self-energy MBPT operator $\Sigma$.) Excitations from the core will have to be included into calculation of the polarizability of the core. This corresponds to working with the non-mixed basis $\Phi_{k}$.

The independence on mixing explains why pure two-valence $a b$ initio calculations of polarizabilities give good results for $\mathrm{Yb}$ despite its complicated structure. This should be true not only for polarizabilities but also for two-photon transition amplitudes, parity non-conservation, etc., and not only for Yb but for some other atoms as well. 


\section{RESULTS AND DISCUSSION}

\section{A. Static polarizabilities}

Our computed static polarizabilities of the clock states are presented in Table III. There we tabulate values obtained in various approximations and we also compile results from the literature. Below we analyze our results and estimate theoretical uncertainties.

The first line of Table III lists the results of pure ab initio calculations, next line gives the results obtained when energies are fitted as explained in Section [I] Then, to gauge the accuracy of the calculations, we introduce various corrections to the polarizability of the ground state based on available experimental data. In the third line of the Table we correct $\alpha_{0}$ to reproduce the experimental value for the magic frequency [9, 15]. This correction will be explained in the following section. The fourth value (135.2) is obtained by replacing the dominant ab initio contribution by semi-empirical values. This corresponds to changing from the unmixed $\Phi_{1}, \Phi_{2}$ basis to the mixed $\Psi_{1}, \Psi_{2}$ basis (see Section III for details). Specifically, we use the value 4.148 a.u. [16] for the $\left\langle 6 s^{2}{ }^{1} \mathrm{~S}_{0}\|d\| 6 s 6 p{ }^{1} \mathrm{P}_{1}\right\rangle$ transition amplitude and $\delta \alpha=24(4)$ [24] for the contribution of the excitations from the $4 f$ core subshell. The resulting value is smaller than both $a b$ initio results (with and without fitting) consistent with our observation made at the end of Section III. Finally, we rescale $\alpha(0)$ of the ground state to fit the experimental value for the van der Waals coefficient $C_{6}[5]$. Since $C_{6}$ is simply obtained by integrating $\alpha(i \omega)^{2}$, Eq. (6) , and both the integral and the static polarizability are dominated by the principal transition, the uncertainties of $\alpha(0)$ and of $C_{6}$ are correlated (see a detailed discussion in Ref. 36 ). The final value for the ground state and its uncertainty, 141(6), are chosen to cover the spread of our numbers in the Table.

Now we turn to the polarizability of the ${ }^{3} \mathrm{P}_{0}^{o}$ state. The central point of our final value is chosen to coincide with the fitted value. Choosing the lower calculated value as the central point is justified by the fact that there is a correction due to excitations for $4 f$ and this correction is negative (see the end of Section [II for details). The value of this correction must be smaller than that for the ground state because corresponding states with excitations from If are higher in the spectrum and closer to the neighboring two-electron states of the same symmetry. Notice that the polarizabilities of both clock states end up having a comparable fractional accuracy. Qualitatively, this may be explained that while the convergence with respect to increasing basis is slower for the ${ }^{3} \mathrm{P}_{0}^{o}$ state, calculations for the ${ }^{1} \mathrm{~S}_{0}$ state are affected stronger by the core-excited states.

Results of other calculations and semi-empirical analyses are presented in Table III for comparison. For the ground state note a good agreement with the value of Ref. 24], $\alpha(0)=136.4(4.0)$, derived using only experimental data. The value $\alpha(0)=143$ recommended by the same authors is also consistent with our result.

The result for the ground-state polarizability is in a disagreement with previous calculations [19, 20, 21]. As discussed in Section III the disagreement for ${ }^{1} \mathrm{~S}_{0}$ comes from the inconsistent use of experimental matrix element for the principal transition in these works. As for the ${ }^{3} \mathrm{P}_{0}^{o}$ state, the present result and that of Ref. [21] differ by about 2 standard deviations and in experimental work this level of agreement between two values would be acceptable.

Finally, let us emphasize that evaluating accuracy of theoretical calculations is a non-trivial exercise and due to the lack of high-accuracy experimental data for the ${ }^{3} P_{0}^{o}$ state, the uncertainty in Ref. [21] was estimated as a half of the difference between pure $a b$ initio result and the result obtained with the fitting of the energy. This may be an unreliable estimate. Suppose we carry out a similar analysis based on the present calculations for the ground state. Fitting energy in $\Sigma_{1}$ moves our ab initio result, 138.9, to 145.7, so using prescription of Ref. [21] would yield 145.7(3.4) which is displaced compared to and is as twice as accurate as our final value of 141(6).

\section{B. Magic frequencies}

Magic frequencies are frequencies of the laser field at which the ac Stark shifts of both clock levels are the same so that the frequency of the clock transition is insensitive to the laser intensity. Magic frequencies, $\omega^{*}$, are found from the condition

$$
\alpha_{1 \mathrm{~S}_{0}}\left(\omega^{*}\right)=\alpha_{3} \mathrm{P}_{0}\left(\omega^{*}\right) .
$$

First five calculated magic frequencies for $\mathrm{Yb}$ are presented in Table IV] The first frequency was measured to a high precision [9, 15]. Our ab initio calculations reproduce it with the $1.3 \%$ accuracy. The accuracy for the other frequencies is likely to be worse. This is because the energy denominators for the dynamic polarizabilities (2) are shifted by the frequency of the laser field and the assumption that the difference in energies of the $4 f^{14} 6 s 6 p{ }^{1} P_{1}^{o}$ and $4 f^{13} 5 d 6 s^{2} 6(7 / 2,5 / 2)_{1}^{o}$ states can be neglected becomes less accurate (see Section III for details).

To improve the accuracy of predicting the magic frequencies and corresponding polarizabilities we modify formula (2) in two ways. First, we use experimental data for the dominant terms: $\left\langle 6 s^{2}{ }^{1} \mathrm{~S}_{0}\|d\| 6 s 6 p{ }^{1} \mathrm{P}_{1}\right\rangle=4.148[16]$ and 
TABLE III: Static electric-dipole polarizability $\alpha(0)$ (in a.u.) of the $6 s^{2}{ }^{1} S_{0}$ and $6 s 6 p^{3} P_{0}^{o}$ clock states of Yb atom in various approximations and comparison with literature values.

\begin{tabular}{|c|c|c|}
\hline$\alpha(0),{ }^{1} \mathrm{~S}_{0}$ & $\alpha(0),{ }^{3} \mathrm{P}_{0}^{o}$ & Comment \\
\hline \multicolumn{3}{|c|}{ this work } \\
\hline 138.9 & 315.9 & $a b$ initio with no fitting \\
\hline 145.7 & 301.6 & energies are fitted by rescaling $\hat{\Sigma}_{1}$ \\
\hline 139.1 & & with a correction to $\alpha\left({ }^{1} \mathrm{~S}_{0}\right)$ to fit the magic frequency $\omega=0.0600$ a.u. \\
\hline 135.2 & & using experimental data for dominant contributions \\
\hline 138.8 & & rescaled to fit experimental data[5] for $C_{6}=1932(30)$ a.u. \\
\hline $141(6)$ & $302(14)$ & final \\
\hline \multicolumn{3}{|c|}{ other } \\
\hline 131.6 & & Wang et al [37]; Density functional theory, 1995 \\
\hline 145.3 & & Wang and Dolg [38]; CCSD(T), 1998 \\
\hline 141.7 & & Miller [39]; Density functional theory, 2002 \\
\hline $118(45)$ & $252(25)$ & Porsev et al [19]; CI+MBPT, 1999 \\
\hline $111.3(5)$ & $266(15)$ & Porsev and Derevianko [20, 21]; CI+MBPT, 2006 \\
\hline 154.7 & & Buchachenko et al [40]; $\operatorname{CCSD}(\mathrm{T}), 2006$ \\
\hline $136.4(4.0)$ & & Zhang and Dalgarno [24]; based on experimental data, 2007 \\
\hline 157.3 & & Chu et al [23]; Density functional theory, 2007 \\
\hline 143 & & recommended value by Zhang and Dalgarno [24], 2007 \\
\hline 144.59 & & Sahoo and Das [26]; relativistic coupled-cluster theory, 2008 \\
\hline
\end{tabular}

$\left\langle 6 s^{2}{ }^{1} \mathrm{~S}_{0}\|d\| 4 f^{13} 5 d 6 s^{2}(7 / 2,3 / 2)_{1}^{o}\right\rangle=2$ [41]. Second, we introduce a correction to simulate the effect of other excitations from the $4 f$ core shell,

$$
\Delta \alpha=\frac{A^{2}}{3}\left(\frac{1}{E-\omega}+\frac{1}{E+\omega}\right),
$$

where $E=37414.59 \mathrm{~cm}^{-1}$ is the energy of the nearby state with the excitation from the core and $A$ is a fitting parameter. Its value is chosen to fit experimental magic frequency. The rest of contributions are taken from the $a b$ initio calculations.

The above empirical correction has been introduced for the ground state only. The polarizability of the upper clock state was computed within the CI+MBPT method for the valence contribution and the DHF method for core polarizability.

The values of magic frequencies and corresponding polarizabilities calculated with this empirical correction are listed in the third and forth columns of Table [V] and the ac polarizabilities are shown in Fig [1. We see that the results for the first four magic frequency change little, while those for the fifth magic frequency vary significantly. Still, while difficult to predict, the fifth magic frequency may be of a practical interest for designing better clocks: by contrast to the first four frequencies, the ac polarizability here may be negative (that was the $a b$ initio prediction, but the empirical correction flipped the sign of the polarizability). Because of that, the atoms are trapped at the minima of the laser intensity, which reduces perturbations of the clock frequency [42].

Finally, using the modified formula at $\omega=0$ provides another accuracy test for our predicted static polarizability of the ground state (Section VA). The resulting value, 139.1 a.u. was listed in Table III and used in estimating the accuracy of the calculations.

\section{Black-body radiation shift}

The effect of black-body radiation (BBR) on the frequency of the Yb clock transition is expressed in terms of the static polarizabilities. The relevant clock shift reads (see detailed derivations in Ref. [21])

$$
\delta \nu_{\mathrm{BBR}} \approx-\frac{2}{15}\left(\alpha_{\mathrm{hfs}} \pi\right)^{3} T^{4}\left(\alpha^{3} P_{0}(0)-\alpha_{{ }^{1} S_{0}}(0)\right)
$$

where $T$ is the temperature and $\alpha_{\mathrm{hfs}}$ is the fine-structure constant. With our polarizabilities we find at $T=300$ $\mathrm{K}, \delta \nu_{\mathrm{BBR}}=-1.41(17) \mathrm{Hz}$ which is in good agreement with the result, $\delta \nu_{\mathrm{BBR}}=-1.34(13) \mathrm{Hz}$, of Ref. [21] despite our significant differences in values of individual polarizabilities. The agreement is fortuitous, as both polarizabilities of Ref. [21] are smaller, most of the shift canceling out while taking the difference in Eq.(23). Note, however, 


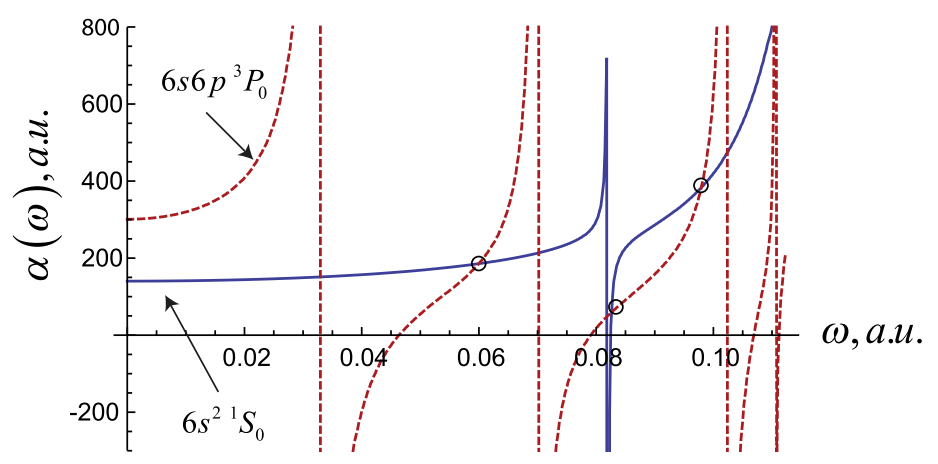

FIG. 1: (Color online) Dynamic polarizabilities of the two clock levels. "Magic" conditions occur when the two polarizabilities intersect off resonance. These are marked by small circles on the plot.

TABLE IV: Magic wavelengths for the $6 s^{2}{ }^{1} S_{0} \rightarrow 6 s 6 p^{3} P_{0}^{o}$ transition in Yb and values of dynamic polarizabilities at corresponding frequencies (a.u.).

\begin{tabular}{|c|c|c|c|c|}
\hline \multicolumn{2}{|c|}{$a b$ initio } & \multicolumn{2}{|c|}{ Corrected $^{a}$} & \multirow{2}{*}{$\begin{array}{c}\text { Experiment } \\
\lambda, n \mathrm{n}\end{array}$} \\
\hline$\lambda, \mathrm{nm}$ & $\alpha(\omega)$, a.u. & $\lambda, \mathrm{nm}$ & $\alpha(\omega)$, a.u. & \\
\hline 749.0 & 193 & 759.37 & 186 & $759.355^{b}$ \\
\hline 552.2 & 46 & 551.5 & 60 & \\
\hline 459.3 & 478 & 465.4 & 382 & \\
\hline 413.2 & 1246 & 413.25 & 817 & \\
\hline 359.7 & -742 & $402.55^{c}$ & $365^{c}$ & \\
\hline
\end{tabular}

${ }^{a} \alpha_{1_{\mathrm{S}_{0}}}(\omega)$ is corrected to fit experimental magic frequency

${ }^{b}$ Ref. 15

${ }^{c}$ unreliable

that a somewhat smaller uncertainty reported in [21] is mostly due to overestimating the theoretical accuracy for polarizabilities, especially for the ground state. See Sections $[I I$ and $\mathrm{VA}$ for details.

It is worth emphasizing, that at the time of writing, the BBR correction is the leading uncertainty in the error budget of the lattice clocks [10]. The present calculations demonstrate a difficulty of atomic theory in determining this important correction accurately. We hope that our analysis would motivate a further experimental work, for example, on cryogenically cooled clock chambers to reduce (and ultimately to accurately determine) the BBR shift.

\section{Atom-wall interaction coefficients $C_{3}$ and van der Waals coefficients $C_{6}$}

We calculate the atom-wall interaction constants $C_{3}$ and the van der Waals constant $C_{6}$ for both clock states using the formulae (5) and (6). The results are presented in Table $\nabla$ together with the results of other calculations and measurements for the $C_{6}$ coefficient for the ground state.

For two Yb atoms in their ground states we find $C_{6}\left({ }^{1} S_{0}+{ }^{1} S_{0}\right)=2041$ a.u. (CI+MBPT with rescaled $\Sigma$ to fit the energies). However, from our calculations of the polarizabilities we know that the contributions from the excitations from the $4 f$ core state are likely to make the results smaller. Therefore, we rescaled the central point for $C_{6}\left({ }^{1} S_{0}+{ }^{1} S_{0}\right)$ at twice the rate as for the polarizability of the ground state (i.e., reduced it by $6.5 \%$ ). This moves the the central value to 1909 a.u. Since the $C_{6}$ is obtained as a quadrature of the square of polarizability, we assign a fractional uncertainty to $C_{6}$ as twice as large as that for the static polarizabilities. Note a good agreement of the ground-state $C_{6}$ with recent experimental determinations [5] and with calculations [24]. The results in the Table for $C_{6}\left({ }^{1} S_{0}+{ }^{3} P_{0}\right)$ and $C_{6}\left({ }^{3} P_{0}+{ }^{3} P_{0}\right)$ were obtained in CI+MBPT with rescaled $\Sigma$ to fit the energies.

Including core polarizability into computation of $C_{6}$ is important. We find that neglecting $\alpha_{\text {core }}(i \omega)$ reduces the final result for $C_{6}$ by about $10 \%$. This is consistent with the earlier observation [27] for heavy alkali-metal atoms.

Atom-wall interaction coefficients $C_{3}$ for the clock states were obtained in CI+MBPT with rescaled $\Sigma$ to fit the energies; these are also listed in the Table $\mathrm{V}$. Notice, that the difference in $C_{3}$ coefficients for the clock states was 
computed by us earlier in Ref. 12]. While forming the difference the contribution of core polarizability cancels out. For individual states, this contribution is substantial. For example, for the ground state result is increased from 2.09 to 3.34 a.u. due to $\alpha_{\text {core }}(i \omega)$. As a large fraction of $C_{3}$ value is accumulated due to the core excitations, assigning error to $C_{3}$ is difficult For example, to estimate error in $C_{3}$ for simpler alkali-metal atoms in Ref. [27], results of two methods of computing $C_{3}$, (i) via the integral of $\alpha(i \omega)$ and (ii) by computing expectation value of some two-body dipole-dipole interaction were compared. A highly-technical evaluation of the expectation value of two-body operator is beyond the scope of the present paper and we do not assign uncertainties to $C_{3}$. We expect that the errors in $C_{3}$ are unlikely to exceed $50 \%$.

TABLE V: Atom-wall interaction coefficients $C_{3}$ and van der Waals coefficients $C_{6}$ (a.u.) for the $6 s^{2}{ }^{1} S_{0}$ and $6 s 6 p^{3} P_{0}^{o}$ states of $\mathrm{Yb}$.

\begin{tabular}{|c|c|c|c|c|}
\hline \multicolumn{3}{|c|}{ Value } & \multicolumn{2}{|c|}{ Comment } \\
\hline \multicolumn{5}{|c|}{ Atom-wall interaction } \\
\hline$C_{3}$ & ${ }^{1} S_{0}$ & 3.34 & Calc. & this work \\
\hline$C_{3}$ & ${ }^{3} P_{0}^{o}$ & 3.68 & Calc. & this work \\
\hline \multicolumn{5}{|c|}{ Atom-atom interaction } \\
\hline$C_{6}$ & ${ }^{1} S_{0}+{ }^{1} S_{0}$ & $1909(160)$ & Calc. & this work \\
\hline & & $2300(250)$ & Exp. & Enomoto et al [17] \\
\hline & & $1932(35)$ & Exp. & Kitagawa et al [5] \\
\hline & & $2400-2800$ & Calc. & Buchachenko et al [40] \\
\hline & & 2292 & Calc. & Chu et al [23] \\
\hline & & 2062 & Calc. & Zhang and Dalgarno [24] \\
\hline$C_{6}$ & ${ }^{3} P_{0}^{o}+{ }^{3} P_{0}^{o}$ & $3886(360)$ & Calc. & this work \\
\hline$C_{6}$ & ${ }^{1} S_{0}+{ }^{3} P_{0}^{o}$ & $2709(338)$ & Calc. & this work \\
\hline
\end{tabular}

\section{CONCLUSION}

We calculated polarizabilities of the clock states of $\mathrm{Yb}$ with the accuracy of about $5 \%$. The substantial disagreement with previous calculations for the ground state polarizability was explained and resolved. We also computed the first four magic frequencies of the lattice laser field, the effect of black-body radiation on the frequency of the clock transition, the $C_{3}$ atom-wall interaction constants and the $C_{6}$ van der Waals coefficients for both clock states. Polarizabilities, the first magic frequency and the $C_{6}$ coefficient for the ground state are in a good agreement with the most accurate calculations and measurements. The presented data may be of interest for designing better clocks, applications of the clocks in studying atom-wall interaction and quantum information processing, and quantifying molecular potentials for ultracold collision studies.

\section{Acknowledgments}

The authors are grateful to S. G. Porsev and A. Dalgarno for useful discussions. The work was supported in part by the Australian Research Council and U.S. National Science Foundation.

[1] K. Tsigutkin, D. Dounas-Frazer, A. Family, J. E. Stalnaker, V. V. Yashchuk, and D. Budker, Observation of a large atomic parity violation effect in ytterbium (2009), arXiv.org:0906.3039, URL http://www.citebase.org/abstract?id=oai:arXiv.org:0906.3039

[2] Y. Takahashi et al., in CP Violation and its Origins, edited by K. Hagiwara (KEK, Tsukuba, 1997).

[3] Y. Takasu, K. Maki, K. Komori, T. Takano, K. Honda, M. Kumakura, T. Yabuzaki, and Y. Takahashi, Phys. Rev. Lett. 91, 040404 (2003).

[4] K. Enomoto, K. Kasa, M. Kitagawa, and Y. Takahashi, Phys. Rev. Lett. 101, 203201 (pages 4) (2008), URL http://link.aps.org/abstract/PRL/v101/e203201

[5] M. Kitagawa, K. Enomoto, K. Kasa, Y. Takahashi, R. C. o, P. Naidon, and P. S. Julienne, Physical Review A (Atomic, Molecular, and Optical Physics) 77, 012719 (pages 8) (2008), URL http://link.aps.org/abstract/PRA/v77/e012719.

[6] T. Fukuhara, S. Sugawa, Y. Takasu, and Y. Takahashi, Phys. Rev. A 79, 021601 (pages 4) (2009), URL http://link.aps.org/abstract/PRA/v79/e021601 
[7] H. Katori, M. Takamoto, V. G. Pal'chikov, and V. D. Ovsiannikov, Phys. Rev. Lett. 91, 173005 (2003).

[8] S. G. Porsev, A. Derevianko, and E. N. Fortson, Phys. Rev. A 69, 021403(R) (2004).

[9] Z. W. Barber, C. W. Hoyt, C. W. Oates, L. Hollberg, A. V. Taichenachev, and V. I. Yudin, Phys. Rev. Lett. 96, 083002 (2006)

[10] N. D. Lemke, A. D. Ludlow, Z. W. Barber, T. M. Fortier, S. A. Diddams, Y. Jiang, S. R. Jefferts, T. P. Heavner, T. E. Parker, and C. W. Oates, Phys. Rev. Lett. 103, 063001 (pages 4) (2009), URL http://link.aps.org/abstract/PRL/v103/e063001

[11] V. V. Flambaum and V. A. Dzuba, Can. J. Phys. 87, 25 (2009).

[12] A. Derevianko, B. Obreshkov, and V. A. Dzuba (2009), arxiv.org:0905.4780, URL http://arxiv.org/abs/0905.4780.

[13] D. Hayes, P. S. Julienne, and I. H. Deutsch, Phys. Rev. Lett. 98, 070501 (pages 4) (2007), URL http://link.aps.org/abstract/PRL/v98/e070501

[14] A. J. Daley, M. M. Boyd, J. Ye, and P. Zoller, Phys. Rev. Lett. 101, 170504 (pages 4) (2008), URL http://link.aps.org/abstract/PRL/v101/e170504

[15] Z. W. Barber, J. E. Stalnaker, N. D. Lemke, N. Poli, C. W. Oates, T. M. Fortier, S. A. Diddams, L. Hollberg, C. W. Hoyt, A. V. Taichenachev, et al., Phys. Rev. Lett. 100, 103002 (pages 4) (2008), URL http://link.aps.org/abstract/PRL/v100/e103002

[16] Y. Takasu, K. Komori, K. Honda, M. Kumakura, T. Yabuzaki, and Y. Takahashi, Phys. Rev. Lett. 93, 123202 (2004).

[17] K. Enomoto, M. Kitagawa, K. Kasa, S. Tojo, and Y. Takahashi, Phys. Rev. Lett. 98, 203201 (pages 4) (2007), URL http://link.aps.org/abstract/PRL/v98/e203201

[18] N. Poli, Z. W. Barber, N. D. Lemke, C. W. Oates, L. S. Ma, J. E. Stalnaker, T. M. Fortier, S. A. Diddams, L. Hollberg, J. C. Bergquist, et al., Physical Review A (Atomic, Molecular, and Optical Physics) 77, 050501 (pages 4) (2008), URL http://link.aps.org/abstract/PRA/v77/e050501

[19] S. G. Porsev, Yu. G. Rakhlina, and M. G. Kozlov, Phys. Rev. A 60, 2781 (1999).

[20] S. G. Porsev and A. Derevianko, Zh. Eksp. Teor. Fiz. 129, 227 (2006), [Sov. Phys.-JETP 102 195, (2006)].

[21] S. G. Porsev and A. Derevianko, Phys. Rev. A 74, 020502(R) (2006).

[22] V. D. Ovsiannikov, V. G. Pal'chikov, A. V. Taichenachev, V. I. Yudin, H. Katori, and M. Takamoto, Phys. Rev. A 75, 020501 (pages 4) (2007), URL http://link.aps.org/abstract/PRA/v75/e020501

[23] X. Chu, A. Dalgarno, and G. C. Groenenboom, Physical Review A (Atomic, Molecular, and Optical Physics) 75, 032723 (pages 4) (2007), URL http://link.aps.org/abstract/PRA/v75/e032723.

[24] P. Zhang and A. Dalgarno, J. Phys. Chem. A 111, 12471 (2007), ISSN 1089-5639, URL http://dx.doi.org/10.1021/jp0750856

[25] P. Zhang and A. Dalgarno, Molecular Physics: An International Journal at the Interface Between Chemistry and Physics 106, 1525 (2008), ISSN 0026-8976, URL http://www.informaworld.com/10.1080/00268970802126608

[26] B. K. Sahoo and B. P. Das, Physical Review A (Atomic, Molecular, and Optical Physics) 77, 062516 (pages 5) (2008), URL http://link. aps.org/abstract/PRA/v77/e062516

[27] A. Derevianko, W. R. Johnson, M. S. Safronova, and J. F. Babb, Phys. Rev. Lett. 82, 3589 (1999).

[28] V. A. Dzuba, V. V. Flambaum, and M. G. Kozlov, Phys. Rev. A 54, 3948 (1996).

[29] V. A. Dzuba and W. R. Johnson, Phys. Rev. A 57, 2459 (1998).

[30] A. Dalgarno and J. T. Lewis, Proc. R. Soc. London, Ser. A 223, 70 (1955).

[31] W. R. Johnson and J. Sapirstein, Phys. Rev. Lett. 57, 1126 (1986).

[32] W. C. Martin, R. Zalubas, and L. Hagan, Atomic Energy Levels - The Rare-Earth Elements (National Bureau of Standards, Washington, 1978).

[33] V. A. Dzuba and J. S. M. Ginges, Phys. Rev. A 73, $032503 \quad$ (pages 8) (2006), URL http://link.aps.org/abstract/PRA/v73/e032503

[34] V. A. Dzuba and V. V. Flambaum, Phys. Rev. A 75, 052504 (2007).

[35] W. R. Johnson, Adv. At. Mol. Opt. Phys. 25, 375 (1988).

[36] A. Derevianko and S. G. Porsev, Phys. Rev. A 65, 053403 (2002).

[37] S. G. Wang, D. K. Pan, and W. H. E. Schwarz, The Journal of Chemical Physics 102, 9296 (1995), URL http://link.aip.org/link/?JCP/102/9296/1

[38] Y. Wang and M. Dolg, Theor. Chem. Acc. 100, 125 (1998).

[39] CRC Handbook of Chemistry and Physics (CRC Press, Boca Raton, FL, 2004), 85th ed.

[40] A. A. Buchachenko, M. gorzata M. Szcześniak, and G. C. asiński, J. Chemi. Phys. 124, 114301 (pages 8) (2006), URL http://link.aip.org/link/?JCP/124/114301/1.

[41] K. B. Blagoev and V. A. Komarovskii, At. Data Nucl. Data Tables 56, 1 (1994).

[42] M. Takamoto, H. Katori, S. I. Marmo, V. D. Ovsiannikov, and V. G. Pal'chikov, Phys. Rev. Lett. 102, 063002 (pages 4) (2009), URL http://link.aps.org/abstract/PRL/v102/e063002 